\title{
Modeling and Simulation of Emergency Service Facilities Location Problem Under Fuzzy Environment
}

\author{
Zixue Guo* and Meiran Qi
}

School of Management, Hebei University, No.180 Wusidong Road. Baoding City, Hebei Province, 071002, China

\begin{abstract}
The emergency service facilities location is one of the most important issues in the emergency management. This study focused on the location set covering problem (LSCP) for emergency service facilities under fuzzy environment. On the basis of defining the signed distance of trapezoidal fuzzy number, the ranking rules of trapezoidal fuzzy number are defined, the model of emergency service facilities location with fuzzy restraints is proposed, and the solution algorithm for the emergency service location based on the ordering rules of trapezoidal fuzzy number is given. Finally, a numerical simulation is used to illustrate the application of the method, which shows that the algorithm is feasible and advantageous.
\end{abstract}

Keywords: Emergency service facility, location model, optimization modeling, simulation, trapezoidal fuzzy number.

\section{INTRODUCTION}

Location planning for emergency service facilities is vital for minimizing losses arising from natural disasters and manmade catastrophic events. In the recent years, large scale emergencies have occurred more frequently, such as the “9.11”terrorist attacks in US (2011), SARS in China (2003), Ocean earthquake and Tsunami in India (2004), Katrina hurricane in US (2005), Wenchuan earthquake in China (2008), and the fatal earthquake in Japan (2011). These catastrophic events have brought huge casualties and property damage. Although these disasters are difficult to predict, yet it can effectively reduce losses brought by the disaster and improve the effect of handling the emergency to establish a valid emergency management system. The emergency service facilities play an important role in emergency management. Emergency service facilities location problem has given rise to the serious concern in emergency management. The scientific and reasonable emergency service facility location decision-making will be beneficial to the disposal of emergency management services.

The facilities location problem is often categorized as covering location problem, p-median problem, p-center problem, etc., while covering location problem also includes the location set covering problem (LSCP) and maximal covering location problem (MCLP). The location set covering problem (LSCP) has been proposed by C. Toregas and C. Revell, who sought a solution for locating the least number of facilities to cover all demand points within the service distance [1]. Church and Revelle introduced a maximal covering location problem (MCLP) that could handle the problem with different populations, demand

\footnotetext{
*Address correspondence to this author at the School of Management, Hebei University, No. 180 Wusidong Road. Baoding City, Hebei Province, 071002, China; Tel: 0086-13463421681; Fax: 0086-312-5079318; E-mail: zixueguo@163.com
}

quality and remoteness of demand points [2]. Since the effectiveness of the facilities will increase when the total distance decreases, Hakimi developed a p-median problem to minimize the total distance between demands and facilities [3]. The p-center problem is a related problem with the LSCP, which seeks the location of p-facilities such that the maximum distance (or time) from any demand point to its nearest facilities is minimized [4].

In more recent years, there exists a fair amount of literature on facilities location problems. Berman and Drezner (2008) presented a P-median problem that handles uncertainty by minimizing the expected cost of serving all demand nodes in the future [5]. Murawski and Church (2009) proposed a model to assume that the established facilities are fixed and the accessibility of demand nodes are to be improved. Their model is called maximal covering network improvement problem which was formulated as an integer-linear programming problem and a case study in Ghana is surveyed [6]. Adriana F. Gabor, Jan-Kees C.W. van Ommeren (2010) proposed a new integer programming formulation for the multilevel facility location problem and a novel 3-approximation algorithm based on LP-rounding [7]. Wen and Iwamura (2008) presented a new a-cost model with fuzzy demands under the Hurwicz criterion. In order to solve this model, the paper proposed a hybrid intelligent algorithm integrated by the simplex algorithm, fuzzy simulations and a genetic algorithm [8]. Batanovic, Petrovic, and Petrovic (2009) suggested maximal covering location problems in networks with uncertainty. They studied problems with equal importance of demand nodes, relative deterministic weights of demand nodes, and linguistic terms for weights of demands [9]. Yang et al. (2007) investigated facility location problems in a fuzzy environment, and designed a chance constrained programming model for the problem [10]. Farzin Taghipourian, Iraj Mahdavi, Nezam Mahdavi-Amiri and Ahmad Makui (2012) presented a fuzzy integer liner programming approach to propose a dynamic virtual hub 
location problem with the aim of minimizing transportation cost in the network [11].

The problem of location planning for emergency service facilities can be classified as a special case of the general facility location problem. Recently, the problem of locating emergency service facilities has received considerable attention. Jia et al. (2006) presented an uncapacitated version of the covering model to locate staging areas in the event of a large-scale emergency. The location of the facilities and the allocation of the demand points to the open facilities are primarily based on distance constraints [12]. Araz, Selim, and Ozkarahan (2007) considered a multi-objective fuzzy goal programming for covering-based emergency vehicle location model. The objective of Araz et al. (2007) is to maximize the population with backup coverage and increasing the service level by minimizing the total travel distance from locations at a distance larger than a prespecified distance standard [13]. Berman and Gavious (2007) presented competitive location models to locate facilities that contain resources required for response to a terrorist attack. They consider the worst-case scenario where the terrorist has the knowledge of the location of the facilities and the State needs to take this into account [14]. Anjali Awasthi, S.S. Chauhan, S.K. Goyal (2011) presented a multi-criteria decision-making approach for location planning for urban distribution centers under fuzzy environment [15].

This paper studied the location problem of emergency service facilities based on the ranking rules of trapezoidal fuzzy number. The remainder of this paper is organized as follows: Section 2 presented the preliminaries of fuzzy set theory. Section 3 described the problem of emergency service facilities location, and constructed the mathematical model of this problem. A new optimization algorithm based on the ranking rules of trapezoidal fuzzy number is introduced in section 4 . In section 5, an example is given for illustrating the use of the model. Conclusions are provided in section 6 .

\section{PRELIMINARIES}

In this section, we briefly review some basic concepts of trapezoidal fuzzy numbers and some existing methods for ranking fuzzy numbers $[16,17]$.

\subsection{Basic Definitions}

Definition 1: A fuzzy set $\tilde{A}$ defined on a universe X may be given as:

$\tilde{A}=\left\{<x, \mu_{\tilde{A}}(x)>\mid x \in X\right\}$,

where $\mu_{\tilde{A}}: X \rightarrow[0,1]$ is the membership function of $\tilde{A}$. The membership value $\mu_{\tilde{A}}(x)$ describes the degree of the element $x \in X$ in $\tilde{A}$.

Definition 2: A fuzzy number $\tilde{A}=[a, b, c, d]$ is said to be a trapezoidal fuzzy number if its membership function is given by:

$$
\mu_{\tilde{A}}(x)=\left\{\begin{array}{cc}
(x-a) /(b-a), & a \leq x<b \\
1, & b \leq x \leq c \\
((x-d) /(c-d), & c<x \leq d \\
0, & \text { otherwise }
\end{array}\right.
$$

\subsection{Arithmetic Operations}

In this section, arithmetic operations between two nonnormal p-norm trapezoidal fuzzy numbers, defined on the universal set of real numbers $\mathrm{R}$, are presented [17].

Assume that $\tilde{A}$ and $\tilde{B}$ are two generalized trapezoidal fuzzy numbers, where $\tilde{A}=\left[a_{1}, b_{1}, c_{1}, d_{1} ; \omega_{1}\right]$, $\tilde{B}=\left[a_{2}, b_{2}, c_{2}, d_{2} ; \omega_{2}\right]$, then, we have

$\tilde{A} \oplus \tilde{B}=\left[a_{1}+a_{2}, b_{1}+b_{2}, c_{1}+c_{2}, d_{1}+d_{2} ; \min \left(\omega_{1}, \omega_{2}\right)\right]$

$\tilde{A}-\tilde{B}=\left[a_{1}-d_{2}, b_{1}-c_{2}, c_{1}-b_{2}, d_{1}-a_{2} ; \min \left(\omega_{1}, \omega_{2}\right)\right]$

$k \tilde{A}=\left\{\begin{array}{c}{\left[k a_{1}, k a_{2}, k a_{3}, k a_{4}\right], k \geq 0} \\ {\left[k a_{4}, k a_{3}, k a_{2}, k a_{1}\right], k<0}\end{array}\right.$, for each $k \in R$

\subsection{Ranking Function}

Definition 3: Assume that $\tilde{A}=[a, b, c, d]$ is a trapezoidal fuzzy number. Let $(\tilde{A})_{\alpha}$ be its intervals of confidence at the level of presumption a (i.e., a-cut):

$(\tilde{A})_{\alpha}=\left\{x \mid \mu_{\tilde{A}} \geq \alpha\right\}, \quad \alpha \in[0,1]$.

Then $(\tilde{A})_{\alpha}=\left\{x \mid \mu_{\tilde{A}} \geq \alpha\right\}$ is an interval number, and $(\tilde{A})_{\alpha}=[a+(b-a) \alpha, \quad d+(c-d) \alpha]$.

Let $(\tilde{A})_{\alpha}^{L}=a+(b-a) \alpha,(\tilde{A})_{\alpha}^{R}=d+(c-b) \alpha$, then $\alpha$-cut of $\tilde{A}$ becomes

$(\tilde{A})_{\alpha}=\left[(\tilde{A})_{\alpha}^{L}, \quad(\tilde{A})_{\alpha}^{R}\right]$,

where $(\tilde{A})_{\alpha}^{L}=a+(b-a) \alpha$ denotes the left points of $(\tilde{A})_{\alpha}$, $(\tilde{A})_{\alpha}^{R}=d+(c-b) \alpha$ denotes the right points of $(\tilde{A})_{\alpha}$.

Definition 4: For each $a, 0 \in R$, define the signed distance of a by $d^{*}(a, 0)=a$.

Definition 5: For interval $(\tilde{A})_{\alpha}=\left[(\tilde{A})_{\alpha}^{L},(\tilde{A})_{\alpha}^{R}\right]$, define the signed distance of $(\tilde{A})_{\alpha}$ as follows:

$$
\begin{aligned}
& d^{*}\left[(\tilde{A})_{\alpha}^{L},(\tilde{A})_{\alpha}^{R}\right]=\frac{1}{2}\left[d^{*}\left((\tilde{A})_{\alpha}^{L}, 0\right)+d^{*}\left((\tilde{A})_{\alpha}^{R}, 0\right)\right] \\
& =\frac{1}{2}[(a+d+(b+c-a-d) \alpha] .
\end{aligned}
$$

Definition 6: If $\tilde{A}=\left[\gamma_{1}, m_{1}, m_{2}, \gamma_{2}\right]$ is a trapezoidal fuzzy number, define the signed distance of $\tilde{A}$ as follows:

$$
d(\tilde{A})=\int_{0}^{1} d\left[(\tilde{A})_{\alpha}^{L},(\tilde{A})_{\alpha}^{R}\right] d \alpha=\frac{1}{4}[a+b+c+d] .
$$


Definition 7: Assume that $\tilde{A}, \tilde{B}$ are two trapezoidal fuzzy numbers, where $\tilde{A}=\left[a_{1}, b_{1}, c_{1}, d_{1}\right], \tilde{B}=\left[a_{2}, b_{2}, c_{2}, d_{2}\right]$, define the ranking of $\tilde{A}, \tilde{B}$ by saying:

$\tilde{A}<\tilde{B}$ iff $d(\tilde{A})<d(\tilde{B}) ;$

$\tilde{A}=\tilde{B}$ iff $d(\tilde{A})=d(\tilde{B})$.

\section{DESCRIPTION AND MODEL OF THE PROBLEM}

\subsection{Problem Description}

Different from the traditional problem of locating facilities, which mainly aims at the cost minimization, the primary goal of the emergency service location problem is to quickly respond to the urgent needs of the affected people. Once the rescue demand is received, the rescue equipment and relief personnel must be transported efficiently to the affected areas, especially in the crucial rescue period. That is, quick response is the key for locating emergency service facilities.

Location set covering problem (LSCP) is one of the covering location problems, which aims at locating the least number of facilities that are required to cover all demand points. Given a cover standard for a service, such as traveltime $\lambda$, one of the key assumptions of the LSCP is that a demand point is assumed to be fully covered if located within a travel-time $\lambda$ from a facility and noted covered if it is farther than $\lambda$ away from any facility.

In this paper, we considered a given geographical area, and assumed that there are $\mathrm{m}$ demand points and $\mathrm{n}$ possible candidate location points in the area. Let $D=\left\{D_{1}, D_{2}, \cdots, D_{m}\right\}$ be the set of demand point, and $S=\left\{S_{1}, S_{2}, \cdots, S_{n}\right\}$ be the set of candidate locations where service facilities may be located. $\lambda$ denoted the threshold value of travel time, then a candidate location $j$ can provide service to a demand point $i$ only if the traveling time $\tilde{t}_{i j}$, between demand point $i$ and candidate location $j$, is within the given threshold value $\lambda$. We also assumed that the construction cost of all candidate location points is the same. Given the service request of the entire geographical area, the problem is to decide where to locate the emergency service station to cover all demand points with the least possible number of facilities.

\subsection{Mathematical Model}

Here, it has been assumed that the travel times between demand point $i$ and candidate location $j$ are uncertain which are presented as a trapezoidal fuzzy numbers $\tilde{t}_{i j}$ :

$\tilde{t}_{i j}=\left(t_{i j a}, t_{i j b}, t_{i j c}, t_{i j d}\right)$.

Before introducing the problem formation, decision variables and parameters are presented as follows:

Problem parameters $n$ Number of possible candidate facilities;

$m$ Number of demand points;

$\tilde{t}_{i j}$ Travel time between nodes $i$ and $j$;

$\lambda$ Coverage radius;

$N_{i}$ The set of all candidate locations that can cover the demand point $i$, let $N_{i}=\left\{j \mid \tilde{t}_{i j} \leq \lambda\right\}(i=1,2, \cdots, m)$, where $\tilde{t}_{i j} \leq \lambda$ is a fuzzy constrain.

Decision variable $x_{j}=\left\{\begin{array}{ll}1, & \text { if a facility is established in candidate location } j \\ 0, & \text { otherwise }\end{array}\right.$.

Now the problem of locating emergency service facilities under fuzzy environment could be stated as follows:

$$
\begin{aligned}
& \min \quad z=\sum_{j=1}^{n} x_{j} \\
& \text { s.t. } \quad \sum_{j \in N_{i}} x_{j} \geq 1(i=1,2, \cdots, m) \\
& x_{j} \in\{0,1\} \quad j=1,2, \cdots, n
\end{aligned}
$$

Here, the objective function (1) is the minimization of the total numbers of emergency service facilities that may be located. Constrains (2) are coverage constrains which ensure that all demand points are covered by a service facility $j \in N_{i}$. Constrains (3) define the $0-1$ nature of the decision variables.

\section{ALGORITHM OF THE MODEL}

To solve the above fuzzy mathematical programming (1)(3), we used firstly the ranking rule to transform the fuzzy constraint $\tilde{t}_{i j} \leq \lambda$ into the deterministic constraint:

$\frac{1}{4}\left(t_{i j a}+t_{i j b}+t_{i j c}+t_{i j d}\right) \leq \lambda$

Now the covered set $N_{i}=\left\{j \mid \tilde{t}_{i j} \leq \lambda\right\}$ becomes $N_{i}^{\prime}$ :

$N_{i}^{\prime}=\left\{j \mid \frac{1}{4}\left(t_{i j a}+t_{i j b}+t_{i j c}+t_{i j d}\right) \leq \lambda\right\}$

Let (4) into the original model, the fuzzy mathematics programming (1)-(3) is formulated as:

$$
\begin{aligned}
& \min \quad Z=\sum_{j=1}^{n} x_{j} \\
& \text { s.t. } \quad \sum_{j \in N_{i}^{\prime}} x_{j} \geq 1 \quad(i=1,2, \cdots, m) \\
& x_{j} \in\{0,1\} \quad j=1,2, \cdots, n
\end{aligned}
$$

The above problem (5)-(7) is a linear programming, which can be solved by simplex method. 
The main steps of this algorithm are stated as follows:

Step 1: Define the covered set $N_{i}=\left\{j \mid \tilde{t}_{i j} \leq \lambda\right\}$, which denotes all the candidate facilities that can covered by the demand point $i$.

Step 2: Compute the signed distance $d\left(\tilde{t}_{i j}\right)$ of trapezoidal fuzzy number $\tilde{t}_{i j}$, and obtain the new covered set $N_{i}^{\prime}=\left\{j \mid \frac{1}{4}\left(t_{i j a}+t_{i j b}+t_{i j c}+t_{i j d}\right) \leq \lambda\right\}$.

Step 3: Let $N_{i}^{\prime}$ be the original problem (1)-(3), with which we can get the linear programming problem (5)-(7).

Step 4: Use simplex method to solve the linear programming problem (5)-(7), we can obtain the optimal plan for locating emergency service facilities.

\section{NUMERICAL SIMULATION}

In this selection, we will show the effectiveness of the model and algorithm by the following numerical example.

Now let us consider a city in China that plans to locate some fire stations within the urban district. Assume that there are 6 demand points and 5 candidate facilities in the urban area. The travel is denoted by time $\tilde{t}_{i j}$ between demand point $i$ and candidate facility $j$, which are trapezoidal fuzzy numbers. According to the standards of the Chinese Ministry of public security, the principle of locating fire stations is that the fire station can reach the area edge within 5 minutes after receiving the dispatched instruction. So we set coverage radius $\lambda=5$ minutes. The fuzzy travel time $\tilde{t}_{i j}$ from facility $j$ to demand point $i, i=1,2, \cdots, 6, j=1,2, \cdots, 5$ are given in Table $\mathbf{1}$.

Table 1. Travel time from fire station to demand point.

\begin{tabular}{|c|c|c|c|}
\hline & $\boldsymbol{D}_{\mathbf{1}}$ & $\boldsymbol{D}_{\mathbf{2}}$ & $\boldsymbol{D}_{\mathbf{3}}$ \\
\hline \hline$S_{1}$ & {$[3,4,6,7]$} & {$[2,3,5,6]$} & {$[4,5,6,7]$} \\
\hline$S_{2}$ & {$[6,8,12,14]$} & {$[4,7,11,14]$} & {$[2,3,5,6]$} \\
\hline$S_{3}$ & {$[4,6,10,12]$} & {$[4,5,9,11]$} & {$[4,5,7,9]$} \\
\hline$S_{4}$ & {$[4,6,8,10]$} & {$[7,9,13,15]$} & {$[6,8,11,13]$} \\
\hline$S_{5}$ & {$[1,2,4,5]$} & {$[2,3,5,6]$} & {$[1,2,4,6]$} \\
\hline & $\boldsymbol{D}_{4}$ & $\boldsymbol{D}_{\mathbf{5}}$ & $\boldsymbol{D}_{\mathbf{6}}$ \\
\hline \hline$S_{1}$ & {$[4,6,8,9]$} & {$[1,2,4,5]$} & {$[1,2,3,4]$} \\
\hline$S_{2}$ & {$[5,6,8,10]$} & {$[1,2,5,8]$} & {$[1,3,4,5]$} \\
\hline$S_{3}$ & {$[2,3,5,7]$} & {$[4,5,8,10]$} & {$[4,5,7,8]$} \\
\hline$S_{4}$ & {$[4,5,7,8]$} & {$[2,3,5,6]$} & {$[1,2,4,5]$} \\
\hline$S_{5}$ & {$[2,3,4,7]$} & {$[4,5,7,8]$} & {$[5,8,9,12]$} \\
\hline
\end{tabular}

The formulation of the fire station location problem is:

$$
\min Z=\sum_{j=1}^{5} x_{j}
$$

s.t. $\quad \sum_{j \in N_{i}} x_{j} \geq 1 \quad(i=1,2, \cdots, 6)$

$x_{j} \in\{0,1\} \quad j=1,2, \cdots, 5$

According to Table 1, we can get the signed distance $d\left(\tilde{t}_{i j}\right)$ of the trapezoidal fuzzy number (the results are given in Table 2).

Table 2. The signed distance of travel time from fire station to demand point.

\begin{tabular}{|c|c|c|c|}
\hline & $\boldsymbol{D}_{\mathbf{1}}$ & $\boldsymbol{D}_{\mathbf{2}}$ & $\boldsymbol{D}_{\mathbf{3}}$ \\
\hline \hline$S_{1}$ & 5 & 4 & 5.5 \\
\hline$S_{2}$ & 10 & 9 & 4 \\
\hline$S_{3}$ & 8 & 7.25 & 6.25 \\
\hline$S_{4}$ & 7 & 11 & 9.25 \\
\hline$S_{5}$ & 3 & 4 & 3.25 \\
\hline & $\boldsymbol{D}_{4}$ & $\boldsymbol{D}_{\mathbf{5}}$ & $\boldsymbol{D}_{\mathbf{6}}$ \\
\hline \hline$S_{1}$ & 6.75 & 3 & 2.5 \\
\hline$S_{2}$ & 7.25 & 4 & 3.25 \\
\hline$S_{3}$ & 4.25 & 6.75 & 6 \\
\hline$S_{4}$ & 6 & 4 & 3 \\
\hline$S_{5}$ & 4 & 6 & 8.5 \\
\hline
\end{tabular}

The $d\left(\tilde{t}_{i j}\right)$ values from Table $\mathbf{2}$ are used to fuzzy constrain $\tilde{t}_{i j} \leq \lambda$, the mathematical model (8) (10) becomes:

$\min z=x_{1}+x_{2}+x_{3}+x_{4}+x_{5}$

s.t. $x_{1}+x_{5} \geq 1$,

$x_{2}+x_{5} \geq 1$

$x_{3}+x_{5} \geq 1$,

$x_{1}+x_{2}+x_{4} \geq 1$,

$x_{j} \in\{0,1\} \quad j=1,2, \cdots, 5$

The above problem can be solved by the simplex method, its optimal solution is $x_{2}=1, x_{5}=1$, and the optimal value $z^{*}=2$. Therefore, the candidate fires $S_{2}$ and $S_{5}$ are selected as the best location for implementing the fire station for this urban district.

\section{CONCLUSION}

The location problem in emergency service facilities is a complicated, systemic and cross-subject problem. The scientific and reasonable location decision-making of emergency facilities will affect the disposal results of emergency. The fuzzy location model for emergency service facilities proposed in this paper can capture different 
decision-making problems such as the fire station, emergency treatment center etc. This research can provide theoretical reference for optimizing an emergency service facilities system in an urban district.

\section{CONFLICT OF INTEREST}

The authors confirm that this article content has no conflict of interest.

\section{ACKNOWLEDGEMENTS}

This paper is supported by the National Social Science Foundation of China (No. 11BGL089), the Soft Science Foundation of Hebei province of China (No.114572154), the Humanities and social science introduce talents fund of Hebei University of China (No.1009117).

\section{REFERENCES}

[1] C. Toregas, and C. Revelle, "Optimal location under time or distance constrains", Paper of the Regional Science Association, vol. 28, pp. 133-143, 1972.

[2] R. Church, and C. ReVelle, "The maximal covering location problem", Papers of the Regional Science Association, vol. 32, pp.101-118, 1974

[3] S.L. Hakimi, "Optimal location of switching centers and the absolute centers and medians of a graph", Operations Research, vol. 12 , pp. 450-459, 1964.

[4] R.S. Garfinkel, A.W. Neebe, and M.R. Rao, "Them-center problem: Minimax facility location", Management Science, vol. 23, pp. 1133-1142, 1977.

[5] O. Berman, and Z. Drezner, "The P-median problem under uncertainty", European Journal Operational Research, vol. 189, pp. 19-30, 2008.
[6] L. Murawski, and R.L. Church, "Improving accessibility to rural health services: The maximal covering network improvement problem", Socio-Economic Planning Sciences, vol. 43, pp. 102$110,2009$.

[7] A.F. Gabor, and J.-K. C.W. van Ommeren, "A new approximation algorithm for the multilevel facility location problem", Discrete Applied Mathematics, vol. 158, pp. 453-460, 2011.

[8] M. Wen, and K. Iwamura, "Fuzzy facility location-allocation problem under the Hurwicz criterion", European Journal Operational Research, vol. 184, pp. 627-635, 2008.

[9] V. Batanovic, D. Petrovic, and R. Petrovic, "Fuzzy logic based algorithms for maximum covering location problems", Information Sciences, vol. 179, pp. 120-129, 2009.

[10] L.X. Yang, X.Y. Ji, Z.Y. Gao, and K.P. Li, "Logistics distribution centers location problem and algorithm under fuzzy environment", Journal of Computational and Applied Mathematics, vol. 208, pp. 303-315, 2007.

[11] F. Taghipourian, I. Mahdavi, N. Mahdavi-Amiri, and A. Makui, "A fuzzy programming approach for dynamic virtual hub location problem", Applied Mathematical Modelling, vol. 36, pp. 32573270, 2012.

[12] H. Jia, F. Ordónez, and M.M. Dessouky, “A modeling framework for facility location of medical services for large-scale emergencies", IIE Trans, vol. 39, pp. 41-55, 2006.

[13] C. Araz, H. Selim, and I. Ozkarahan "A fuzzy multi-objective covering-based vehicle location model for emergency services", Computers and Operations Research, vol. 34, pp. 705-726, 2007.

[14] O. Berman, A. Gavious, "Location of terror response facilities", Eur J Opl Res, vol. 177, pp. 1113-1133, 2007.

[15] A. Awasthi, S.S. Chauhan, and S.K. Goyal, "A multi-criteria decision making approach for location planning for urban distribution centers under uncertainty", Mathematical and Computer Modelling, vol. 53, pp. 98-109, 2011.

[16] L.A. Zadeh, "Fuzzy sets", Information and Control, vol. 8, pp. 338 353, 1965.

[17] Chen, S. H., "Ranking fuzzy numbers with maximizing set and minimizing set", Fuzzy Sets and Systems, vol. 17, no. 2, pp. 113129,1985

This is an open access article licensed under the terms of the Creative Commons Attribution Non-Commercial License (http://creativecommons.org/licenses/ by-nc/4.0/) which permits unrestricted, non-commercial use, distribution and reproduction in any medium, provided the work is properly cited. 